Revue des patrimoines

27 | 2015

Le cheval et ses patrimoines (2e partie)

\title{
Degas et le cheval
}

\section{Mariel Oberthur}

\section{OpenEdition}

\section{Journals}

Édition électronique

URL : http://journals.openedition.org/insitu/12389

DOI : 10.4000/insitu.12389

ISSN : 1630-7305

\section{Éditeur}

Ministère de la culture

\section{Référence électronique}

Mariel Oberthur, « Degas et le cheval », In Situ [En ligne], 27 | 2015, mis en ligne le 16 novembre 2015, consulté le 30 avril 2019. URL : http://journals.openedition.org/insitu/12389 ; DOI : 10.4000/ insitu. 12389

Ce document a été généré automatiquement le 30 avril 2019

\section{(c) (i) () $\Theta$}

In Situ Revues des patrimoines est mis à disposition selon les termes de la licence Creative Commons Attribution - Pas d'Utilisation Commerciale - Pas de Modification 4.0 International. 


\title{
Degas et le cheval
}

\author{
Mariel Oberthur
}

1 Bien que Degas (1834-1917) ait exposé de nombreuses fois avec les peintres du groupe impressionniste et fréquenté les cafés du bas de la butte de Montmartre, sa préoccupation n'a pas été de chercher à rendre la lumière et ses effets mais plutôt le mouvement, tout particulièrement dans la représentation du cheval.

\section{La recherche du mouvement dans la représentation du cheval}

2 Si certains historiens et chercheurs tiennent Paul Valéry pour avoir évoqué le premier la relation entre le photographe britannique Eadweard Muybridge et Degas, depuis la publication de son livre Degas Danse Dessin en 1938, ils ont sous-estimé le travail de Degas sur le motif en Normandie. Ils ont surtout passé sous silence les nombreux débats relatés dans la presse quotidienne et les revues spécialisées du monde du cheval qui s'interrogeaient sur la nouveauté apportée par les photographies de Muybridge. Valéry écrit : « Il fut l'un des premiers à étudier les vraies figures du noble animal en mouvement au moyen des photographies instantanées du major Muybridge. D'ailleurs, il aimait et appréciait la photographie ... », mais Degas, comme Henri Rivière, son ami graveur et dessinateur, ne s'est servi d'un appareil que vers 1890 et n'a jamais photographié un cheval ; or, cela faisait plus de trente ans que Degas observait inlassablement et dessinait les mouvements du cheval et ses allures, ce qui lui tenait le plus à cœur.

Le monde des Arts s'interrogeait sur le travail de Muybridge. Le journal Gil Blas ${ }^{1}$, dans son numéro du 29 novembre 1881, relate la séance de présentation des photographies de Muybridge dans le nouvel atelier de Meissonier, en présence de plusieurs personnalités et peintres. Le journal les qualifie de "grotesques » quand elles sont vues séparément mais reconnaît que passées par une lanterne magique, elles donnent l'illusion du mouvement. De même, dans la Revue des Arts décoratifs, le sculpteur ${ }^{2}$ Gustave Debrie examine l'évolution artistique du train arrière du galop et affirme que les artistes ont été déconcertés quand ils ont vu les images de Muybridge le représentant. Degas, depuis près 
de quarante ans, cherchait à rendre les allures, comme l'avaient fait avant lui quelques peintres comme Eugène Fromentin notamment.

4 L'importance des séjours de Degas à Ménil-Hubert-en-Exmes, dans l'Orne, chez son ami Paul Valpinçon, n'a jamais été vraiment prise en considération. Degas est venu chez son ami d'enfance de 1861 à 1904, y faisant de nombreux séjours, de quelques semaines à plusieurs mois. Dans sa correspondance, il parle peu de ses séjours normands si ce n'est pour se plaindre du temps pluvieux ou trop chaud. Ménil-Hubert était un grand domaine propriété de la famille Pinson de Valpinçon, acheté en 1822 par Édouard Valpinçon, le père de Paul, amateur des peintures d'Ingres dont il possédait la fameuse Baigneuse aujourd'hui au Louvre. Degas, dans ce grand château à quelques kilomètres du Haras du Pin pouvait peindre à sa guise : il a représenté sa chambre et le salon du billard; Paul, peintre amateur, lui prêtait son atelier, une petite maison dans le domaine. On connaît aussi une série de six photographies ${ }^{3}$ anonymes, prises lors d'un des derniers séjours, en 1904, représentant Degas seul ou avec la fille de Paul, Hortense, et son mari Jacques Fourchy sur la terrasse devant le château.

Dans la campagne aux alentours, près du Haras du Pin, les prés sont pleins de chevaux élevés pour le travail, le transport, la chasse et la course. Degas aime les chevaux, et son escapade en Bourgogne en 1890 avec son ami Bartholomé le prouve bien, quand il s'inquiète de savoir si le «blanc » qui les emmène pour cette longue randonnée en tilbury a assez d'avoine et s'émerveille du chemin qu'il prend pour rentrer à Paris.

De son vivant, seuls quelques tableaux ont été exposés et peu ont été vendus. Dans son atelier à Paris, il ne montre rien sauf à Bartholomé, à Alexis et Louis Rouart. Ménil-Hubert et le cheval est resté le jardin secret de Degas.

7 La référence dans les Carnets à la revue La Nature a induit en erreur les chercheurs et les historiens. Il est vrai que l'on peut se méprendre sur la manière de travailler de Degas et sa recherche pour rendre avec la plus grande exactitude le mouvement et en particulier les allures du cheval. Si on reprend la chronologie des années 1860 à 1880, il n'est pas sérieux de dire que ce sont les photographies de Muybridge ou de Marey qui ont permis à Degas de représenter les différentes allures du cheval, même s'il fait seulement mention de la revue La Nature 4 à la page 81 du Carnet daté $1878^{5}$ (fig. ${ }^{\circ} \mathbf{1}$ ).

Figure 1 - Edgar Degas. Note manuscrite « Journal : la nature/Victor Masson (année 1878). Carnet 23 p. 81. Paris : Bibliothèque nationale de France. http://gallica.bnf.fr/ark:/12148/ btv1b84387031/f22.item.r=Degas\%20carnet23, p. 81, image $35: 83$ page 2 du carnet 23 .

8 Muybridge essayait depuis 1872 d'analyser et de décomposer les mouvements du cheval au galop grâce à une séquence d'images fixes. L'expérience est annoncée dans La Nature en 1874 mais seulement publiée en 1878 dans ce même journal. Quand on lit l'article du 5 octobre ou celui du 14 décembre 1878, on peut comprendre l'intérêt que Degas pouvait avoir à les lire. La seule mention, sans autre indication, de La Nature ne signifie pas que Degas, comme plusieurs autres artistes de son époque, n'ait pas pris conscience des possibilités offertes par la photographie pour représenter les allures du cheval et peutêtre même s'en soit servi, ce qui serait étonnant car cela faisait déjà plus de quinze ans que Degas allait à Ménil-Hubert et observait les chevaux au pré. L'apport de la photographie reste tardif puisque Étienne-Jules Marey ne consacrait qu'en 1873, dans son ouvrage La Machine animale, illustré par Émile Duhousset vingt-huit pages aux allures du cheval. L'année suivante, Duhousset publiait lui-même un livre sur les allures du cheval, illustré et accompagné de plusieurs planches de ses dessins. 
Il conviendrait plutôt de penser que Degas a feuilleté ou lu chez ses amis Valpinçon, qui avaient probablement une grande bibliothèque, ce qui était publié depuis le XVII ${ }^{e}$ siècle et surtout dans la décennie 1850-1860, tournant important dans l'évolution de la connaissance des chevaux et de l'amélioration des races. À l'époque, nombreux étaient les médecins et surtout les maîtres d'équitation comme le comte d'Aure ${ }^{6}$, Charles de Raabe ${ }^{7}$, Auguste Éloire ${ }^{8}$, qui publiaient des traités pour connaitre le cheval, sa morphologie, ses allures et expliquaient, pour certains, comment les dresser et les monter. Degas, en bon cavalier, a certainement pu se documenter pour approfondir sa recherche du mouvement et il a dû être conforté en lisant en 1885 dans le Journal des Haras l'article sur la locomotion du cheval de Charles de Raabe qui conclut que les photographies de Muybridge montrent la parfaite exactitude des théories du monde du cheval, c'est-à-dire que le cheval termine "chaque pas par la projection, après les foulées des pieds antérieurs; il ne saute pas en longueur après les foulées des pieds postérieurs »"

La méthode de Degas pour exprimer le mouvement est évoquée dans ses croquis et esquisses des Carnets, dans ses dessins et ses cires. Ses premiers dessins de cheval sont pour la plupart des copies de tableaux dans les musées italiens et français, des copies de lithographies d'Alfred de Dreux, des tableaux de Géricault et des moulages du Parthénon de l'École des beaux-arts de Paris. Puis, Degas fait ses premiers dessins et croquis de cheval en Normandie sur le motif dès 1861 et ainsi, au cours des années, il établit un corpus de dessins et croquis.

11 C'est donc bien par le trait et la ligne, comme le lui recommandait Ingres, en dessinant dans ses Carnets dont il ne se séparait pas, que Degas a exprimé le mouvement, cette qualité sensorielle qu'Étienne Souriau, dans La Correspondance des Arts ${ }^{10}$, estime spécifique à la peinture, comme l'espace, la couleur, et c'est l'observation du cheval que Degas a privilégiée, davantage encore que celle des danseuses, des blanchisseuses ou des repasseuses.

\section{Les Carnets}

12 Ils sont au nombre de trente-huit, retrouvés dans l'atelier de Degas après sa mort. Vingtneuf sont conservés à la Bibliothèque nationale de France, deux au musée du Louvre à Paris, un au Metropolitan Museum de New York, un à la Morgan Library à New York, un au J. Paul Getty Museum à Malibu (Californie) et enfin quatre dans des collections privées.

13 C'est seulement dans dix-sept Carnets, utilisés de 1853 à 1886, que peuvent être recensés plus de 270 dessins et croquis dont j'ai établi un corpus pour la Bibliothèque nationale de France qui sera prochainement publié dans mon livre Degas et le cheval, une amitié un lieu une ceuvre ${ }^{11}$.

14 Ces Carnets sont de simples calepins, quelquefois sur papier quadrillé et n'ayant pas tous le même format, quelques-uns pouvant être mis dans une poche, d'autres plus grands, contenant des dessins aquarellés plus aboutis. En étudiant les dessins des Carnets, on comprend parfaitement ce travail de recherche et d'expression du mouvement auquel Degas était confronté.

15 Prenons quelques exemples : dans le Carnet 1 , celui qui contient la « fameuse promenade au Haras du Pin» et qui a été utilisé entre 1859 et 1864, date des premiers séjours en Normandie de Degas. À la page 94 (fig. 2) un dessin de cheval vu de face avec un poitrail bien musclé dont Pierre Besnault ${ }^{12}$ remarque que seul un bon cavalier, homme de cheval 
attentif à l'état de sa monture, peut avoir dessiné un tel cheval et, à la page précédente, un même cheval est vu de profil droit au pas, antérieur et postérieur opposé levés (Carnet 1 p. 93) (fig. 3). Ce mouvement est aussi celui du cheval monté par un jockey dessiné dans le Carnet 12, page 79, que nous retrouvons dans les premiers tableaux Aux courses au départ (daté de 1862, collection privée, États-Unis) ou dans Aux courses, le départ (daté 1862, Fogg Art Museum, Cambridge).

Figure 2 - Edgar Degas. Cheval de face. Carnet 1, p. 94. Bibliothèque nationale de France. http://gallica.bnf.fr/ark:/12148/btv1b84387031/f22.item.r=Degas\%20carnet1, p. 94, carnet $1-10: 106$.

17 Figure 3 - Edgar Degas. Cheval de profil au pas, antérieur et postérieur opposé levés. Carnet 1, p. 93. Bibliothèque nationale de France. Source Gallica.bnf.fr/carnet 19:105.

Dans les Carnets se succèdent au fil des pages, au milieu d'autres sujets d'intérêt de Degas, des têtes de cheval, des postérieurs, des arrière-mains, des antérieurs, des profils droit et gauche. Le cheval est quelquefois harnaché ou monté par un jockey (fig. 4) ou un gentleman-rider. Ce peut aussi être un cheval de trait au travail.

19 Figure 4 - Edgar Degas. Jockey à cheval avec l'annotation: «mettre à Philippe des bottines ", dessin au crayon. Carnet 19, p. 42. Paris, Bibliothèque nationale de France. http://gallica.bnf.fr/ark:/12148/btv1b84387031/f22.item.r=Degas\%20carnet19p.42, image $48: 48$ p. 1.

20 Degas a une très grande acuité visuelle, l'œil commande à sa main et lui permet de saisir le moindre mouvement de l'animal qui par nature ne reste pas immobile et auquel il ne peut demander de le répéter; Degas est obligé de saisir le mouvement du cheval dans l'instant. C'est ainsi qu'il dessine dans la campagne lorsqu'il est au Ménil-Hubert : cheval de dos, immobile (Carnet 19, p. 27 et p. 65) ou se tournant vers la droite ou la gauche (Carnet 6, p. 196 (fig. 5) ou p. 200), cheval se cabrant (Carnet 18, p. 24, Carnet 6 p. 193, (fig. 6) ou pâturant (Carnet 19, p. 35 ou carnet 24, p. 5 ou 11, etc.) et les exemples sont nombreux. La plupart des dessins et croquis sont pris sur le vif en Normandie, de 1861 à 1886, date du dernier Carnet.

21 Figure 5 - Edgar Degas. Cheval vu de dos se tournant. Carnet 6, p. 196. Paris, Bibliothèque nationale de France. http://gallica.bnf.fr/ark:/12148/btv1b84387031/f22.item.r=Degas\% 20carnet6p.196, image $8: 200$, carnet 6 , p. 5 .

22 Figure 6 - Edgar Degas. Cheval vu de dos se tournant. Carnet 6, p. 193. Paris, Bibliothèque nationale de France. http://gallica.bnf.fr/ark:/12148/btv1b84387031/f22.item.r=Degas\% 20carnet6p.193, image $5: 197$, carnet 6, p. 5 .

Dans son travail, Degas est confronté à une difficulté épargnée à Meissonier qui, lui aussi, a la passion de l'exactitude et du détail véridique. Salvador Dali ${ }^{13}$ raconte dans son Hommage à Meissonier que «dans le parc de la somptueuse propriété qu'il s'était fait aménager à Poissy, un train lui permet de rouler confortablement près de cavaliers au galop et de croquer ainsi les mouvements du cheval ».

Degas a un œil photographique. Dans son article «Formes, couleurs et mouvements » publié dans la Gazette des Beaux-Arts ${ }^{14}$ de 1882, Georges Guéroult note que Degas n'avait pas besoin des photographies de Muybridge pour dessiner ou peindre un cheval au galop car il lui suffisait de représenter «l'animal au moment où ses jambes, arrivées à l'extrémité de leur parcours, changent de direction, c'est-à-dire au moment où la vitesse étant nulle, l'image oculaire présente le maximum de netteté ». Il ajoute que les 
photographies de Muybridge sont fausses « oculairement parlant » car elles nous donnent une image nette, au moment où, par suite de sa vitesse et de la persistance des impressions de notre rétine, l'œil humain ne voit et ne verra jamais le cheval au galop comme il le montre dans ses dessins [sic] ses photographies. C'est pour cela que Degas a modelé en cire.

\section{Les modelages en cire}

Pour s'aider dans sa recherche du mouvement et l'enrichir, Degas modèle en cire ou en argile. Combien a-t-il fait de modelages? Nous ne le savons pas. Seulement quinze cires représentant des chevaux et des jockeys, parmi les soixante-quinze retrouvées plus ou moins intactes dans l'atelier après sa mort, ont pu être sauvées. Elles ont émerveillé tous ceux qui ont pu alors les voir. François Thiébault-Sisson se souvient de la conversation qu'il a eue avec Degas, en 1897, à Clermont-Ferrand, plusieurs années avant d'écrire son article dans Le Temps en 1931 :

C'est pour ma seule satisfaction que j'ai modelé en cire bêtes et gens, non pour me délasser de la peinture ou du dessin, mais pour donner à mes peintures et à mes dessins plus d'expression, plus d'ardeur et de vie. Ce sont des exercices pour me mettre en train ; du document sans plus. Rien de tout cela n'est fait pour la vente ${ }^{15}$

et le critique d'ajouter que Degas a commencé à sculpter vers 1867, mais selon nous, plus vraisemblablement déjà plus d'une dizaine d'années auparavant.

Ses sculptures lui servent pour ses peintures, cela parait vraisemblable, car il aurait entendu les critiques du Salon dire en 1866 qu'il ne savait ni dessiner ni peindre un cheval. Degas aurait admis aussi au cours de sa conversation avec Thiébault-Sisson qu'« il y a bien longtemps que je le connais ce métier! Depuis plus de trente ans, je le pratique, non pas à vrai dire, d'une façon régulière, mais de temps à autre, quand cela me chante quand j'en ai besoin. »

28 Les chevaux ont été ses premiers modèles, avant les danseuses. C'est donc très tôt, dans les années 1860 , et même dès ses premiers séjours en Normandie, que Degas fait des modelages en cire dont les dessins des Carnets sont à l'origine. Il est difficile de les dater car Degas n'en parle jamais et nous n'avons aucun repère. L'historien et critique John Rewald, qui a été le premier à les cataloguer, pense que les premiers modelages ont été faits dès 1865, quand Degas a observé les chevaux, et non vers 1881, hypothèse de Charles Millard qui s'appuie sur le fait que Degas les aurait modelés après avoir vu les photographies de Muybridge. C'est oublier que quelques autres artistes contemporains, comme par exemple Ernest Meissonier ou Gustave Moreau ${ }^{16}$, ont fait, eux aussi, des modelages préparatoires à leurs peintures.

Comme ses amis Gustave Moreau et Joseph Cuvelier, Degas a donc entrepris de traduire en volume ses dessins et d'utiliser ses modelages pour sa peinture dès qu'il le souhaite et surtout en a le besoin. Il trouve dans le modelage de la cire, de la plastiline ou de la terre cuite une nouvelle manière, dans le prolongement du dessin, d'exprimer le mouvement, de placer, comprendre et appréhender les différentes allures du cheval.

Degas n'a vraisemblablement pas utilisé que des armatures qu'il pouvait trouver et acheter dans le commerce mais il en a aussi fait lui-même comme l'expliquent Daphné Barbour et Shellye Sturman ${ }^{17}$, à l'appui des radiographies effectuées, notamment celle du Cheval au galop monté par un jockey. 

cherchait déjà, en 1857, à insérer des fils de fer dans un modelage pour le consolider. Outre l'armature, Degas bourre avec du bouchon et du tissu pour ne pas utiliser trop de cire ou de plastiline, qu'il trouvait trop onéreuse. Il est difficile de savoir où Degas se fournissait à cette époque, les noms et adresses de deux fournisseurs «Hutantcire/46 quai de l'Hôtel de Ville » et plus en dessous, sur la même page, "Hocheid, rue Odessa 12/ marchand de terre glaise $»^{18}$, ne figurant que dans le Carnet 2 , utilisé par Degas en 1880-1884.

Par facétie, un trait permanent de son caractère, Degas racontait qu'il travaillait dans son atelier dos à la fenêtre avec un cheval de bois qu'il faisait tourner dans la lumière et qu'il lui fallait retourner aux courses pour dessiner des chevaux. De toute évidence, Degas a fait ses modelages d'après les dessins de ses Carnets et les autres dessins sur feuilles, les gardant secrètement dans son atelier car il n'en parle pas dans ses lettres, et ses amis non plus. après la mort de Degas par Adrien Hébrard ${ }^{19}$, John Rewald ${ }^{20}$, Charles Millard ${ }^{21}$ et Anne Pingeot $^{22}$, on s'aperçoit que soient ainsi illustrées les différentes allures et positions du cheval et du jockey, lesquelles peuvent être amovibles et détachés de leurs montures. C'est pourquoi, pour les amateurs de chevaux, une autre numérotation des modelages peut être proposée.

1 - Cheval de profil à l'arrêt, Hébrard 38, Rewald nIII, Pingeot 47

2 - Jeune cheval de deux ou trois ans, Hébrard 38, Rewald nIII, Pingeot 47

3 - Cheval pur-sang au pas, Hébrard 66, Rewald nV, Pingeot.38.

4 - Cheval de trait, Hébrard 30, Rewald nVII, Pingeot.45

5 - Cheval au pas ou au petit trot de parade, 1870, Hébrard 10, Rewald X, Pingeot.52

6 - Cheval au trot, Hébrard 11, Rewald nIV, Pingeot 39

7 - Cheval au trot les pieds ne touchant pas le sol, Hébrard 49 Rewald nXI, Pingeot 51

8 - Cheval au galop sur le pied gauche, tête ronde, queue en l'air, Hébrard 47, Rewald VI, Pingeot 41

9 - Cheval au galop sur le pied droit, monté par un jockey, Hébrard 25 et 35, Rewald, 194, XIV et XV, Pingeot, 49 et 50

10 - Cheval au galop sur le pied gauche tournant la tête à droite et monté par un jockey, Hébrard 32 et 36, Rewald, 1957, XVII, 18, Pingeot 53-54

11 - Cheval en défense, Hébrard 65 Rewald, 1957, XVI, Pingeot, 40

12 - Cheval s'élevant sur l'obstacle, Hébrard 48, Rewald 1957, IX, Pingeot 43,

13 - Cheval se cabrant, Hébrard 4, Rewald 13, Pingeot, 44

14 - Cheval à l'abreuvoir ou cheval pâturant, Hébrard 13, Rewald II, Pingeot, 42

15 - Cheval tête baissée, bouche ouverte, Hébrard 22, Rewald XII, Pingeot 46

Ces quinze modelages en cire sont conservés en trois lieux : à la National Gallery de Washington, au Virginia Fine Arts Museum, et un seul modelage en cire rouge au musée d'Orsay, Cheval de profil à l'arrêt ${ }^{23}$ (Pingeot 47).

Pour ce modelage, Degas a utilisé de nombreux dessins de profil droit ou gauche, d'avant et d'arrière-main, d'antérieurs et de postérieurs qui sont dans les Carnets utilisés avant 
1865-1870 comme par exemple dans le Carnet 1, p. 93 (voir fig. 3), Carnet 8, p. 58 et 78 ( fig. 7), Carnet 12, p. 49. Degas a repris aussi ce cheval à l'arrêt dans plusieurs autres dessins comme celui à la mine de plomb (musée d'Orsay, RF 43226), le dessin au crayon avec des rehauts de gouache (Boston, Isabella Stewart Garden Museum), celui à la craie et au fusain (Williamstown, Sterling and Francine Clark Art Institute) ou encore celui à la sanguine (Rotterdam, Museum Boijmans Van Beuningen). Dans cette cire, le cheval, toutefois, n'est pas bien placé, son antérieur droit est devant le gauche.

51 Figure 7 - Edgar Degas. Cheval humant l'air. Carnet 8, p. 68, 1861-1884. Paris, Bibliothèque nationale de France. http://gallica.bnf.fr/ark:/12148/btv1b843870 31/f22.item.r=Degas\% 20carnet 8, p. 68, image $26: 74$, carnet 8 page 2 .

52 Si l'on s'en tient à l'allure du galop, il reste trois modelages dont deux chevaux montés par un jockey. Le mouvement n'est pas complet, il manque plusieurs phases de cette allure, soit que les cires ont disparu, soit qu'elles étaient si abîmées que Degas lui-même, à son habitude, en perfectionniste qu'il était, les a détruites ; ce qui complique la datation.

53 Degas a dû faire de nombreux essais pour représenter cette allure difficile. Prenons l'exemple des trois cires conservées à la National Gallery of Art de Washington. Dans son catalogue Edgar Degas sculptures ${ }^{24}$, Suzanne Glover Lindsay ne voit, dans ces trois œuvres, que le caractère grossier de la tête du cheval et de celle du jockey et semble oublier les dessins de Normandie, considérant toujours que ce sont les photographies de Muybridge et Marey qui ont permis à Degas de faire ses modelages. Si les têtes ou le visage ne sont qu'ébauchés, c'est que l'intérêt de Degas était vraiment la recherche du mouvement et non la crinière du cheval ou la casaque flottante du jockey.

54 - Le cheval au galop sur le pied gauche, tête ronde, queue en l'air ${ }^{25}$ est saisi au moment où son antérieur gauche est avancé après avoir soulevé ses postérieurs, juste avant le temps de suspension.

55 - Le Cheval au galop sur le pied droit, monté par un jockey ${ }^{26}$ est dans la troisième phase du galop, à l'instant où il va poser son antérieur droit sur le sol pour finir le mouvement. Le jockey est légèrement penché en avant comme pendant une course, position caractéristique de la monte du XIX ${ }^{\mathrm{e}}$ siècle.

56 - Cheval au galop sur le pied gauche tournant la tête à droite et monté par un jockey ${ }^{27}$. Le temps du galop représenté est encore celui de la troisième phase, juste avant le temps de suspension, antérieurs et postérieurs ne touchant pas le sol et antérieur gauche plus allongé. Degas a bien observé qu'un cheval au galop sur le pied gauche a sa tête à droite. La position du corps du jockey est plus en arrière, ébauchant un mouvement de recul tirant sur les rênes pour arrêter son cheval. Plusieurs dessins de jockey dans cette position se trouvent dans un Carnet utilisé en Normandie en 1861 et dans un autre de 1882. Ce qui laisse penser que la cire a été faite dès les années 1862 et que Degas s'en est servi pour de nombreux tableaux jusqu'en 1889: Jockey (Detroit, Institute of Arts), les peintures Avant la course (Washington, National Gallery of Art, ill.), Aux courses jockeys (musée d'Orsay).

57 À la mort de Degas, ses héritiers et les marchands Durand-Ruel et Vollard décident de faire fondre les modelages en bronze dans les ateliers d'Adrien Hébrard à Paris. Ils sont tirés de 1920 à 1937 par Albino Palazzoloº ${ }^{28}$, le maître fondeur d'Adrien Hébrard, selon une technique proche de la cire perdue permettant de sauver les cires en passant par un modèle en bronze dit bronze-modèle qui a servi de matrice pour le tirage des exemplaires 
subséquents des séries qui faisaient l'objet du contrat d'édition passé entre les héritiers et Hébrard (séries Hébrard).

Il y a plus de dix ans, aux alentours de l'année 2000, ont été retrouvés chez le successeur de la fonderie Valsuani qui avait elle-même repris la suite de la fonderie Hébrard, des plâtres correspondant en nombre et sujet à ceux des séries Hébrard de 1920. Une seule question se pose: ces plâtres ont-ils été faits par Palazzolo avant qu'il ne fasse les bronzes-modèles ${ }^{29}$ ou au moment de la vente des cires originales à Paul Mellon par l'intermédiaire de la galerie Knoedler en 1955, ou encore au moment de la vente des bronzes-modèles en 1976 acquis par Simon Norton par l'intermédiaire de la galerie Lefevre à Londres? Toujours est-il qu'aujourd'hui, les héritiers Degas, réunis en Comité Degas, reconnaissent ces plâtres et les tirages en bronze qui en sont faits ${ }^{30}$. De toute façon, les exemplaires provenant de ces deux tirages posthumes sont des multiples, faits après la mort de Degas, lui qui pourtant avait clairement voulu que ses cires ne soient pas figées en bronze pour « l'éternité ».

\section{Les peintures}

Les peintures, peu exposées et peu connues durant la vie de Degas, sont l'aboutissement de tout son travail de dessin et de modelage. On en compte presque une soixantaine. Les premiers tableaux, ceux de 1861 à 1875 environ - des promenades à cheval, des départs de chasse, des courses sur des hippodromes de l'Orne près du Ménil-Hubert - sont sur toile ou sur bois, surtout peints à l'huile, puis ceux de 1875-1880 à 1905 - chevaux dans des paysages que Degas conserve en mémoire ou imagine et dont il fait des séries - sont au pastel quand sa vue a baissé et s'est troublée.

Des exemples : quand Degas peint en 1862 Jockeys avant le départ (coll. part.) ${ }^{31}$ huile sur toile de 1862, l'un de ses premiers tableaux qui représente un départ dans une forêt, certainement celle de Gouffern, à seulement quelques kilomètres du Ménil-Hubert, il cherche dans ses premiers Carnets plusieurs dessins et trouve le modèle du cheval monté à gauche dans le Carnet 12 p. 79 et dans le 19, p. 42. Pour celui de droite, il puise encore dans le Carnet 19, p. 27 ou 31 et se souvient certainement des copies qu'il a faites d'après Géricault. Il a aussi devant les yeux les modelages de Cheval à l'arrêt ${ }^{32}$ ou de Cheval pur-sang au pas ${ }^{33}$. Quand il peint en 1866 Départ d'une course devant les tribunes ${ }^{34}$ (musée d'Orsay), ce sont des dessins du Carnet 16 (fig. $\left..^{\circ}, \mathbf{9}, \mathbf{1 0}, \mathbf{1 1}\right)$ qu'il prend, ceux qu'il a copiés d'après Géricault ${ }^{35}$ et ceux qu'il a observés en Normandie ${ }^{36}$.

61 Figure 8 - Edgar Degas. Postérieurs, copie partielle de Cinq chevaux vus par la croupe de Géricault et en haut de la page, copie d'un cheval de profil droit de Alfred de Dreux et en bas une tête de jockey. Dessins au crayon. Carnet 16, p. 61, 1859-1869. Paris, Bibliothèque nationale de France. http://gallica.bnf.fr/ark:/12148/btv1b84387031/f22.item.r=Degas\% 20carnet16p.61, image $15: 63$, carnet 16 p. 2.

Figure 9 - Edgar Degas. Arrière-main d'un cheval de profil droit. Esquisse au crayon. Carnet 16, p. 107. Paris, Bibliothèque nationale de France. http://gallica.bnf.fr/ark:/12148/ btv1b84387031/f22.item.r=Degas\%20carnet16p.107, image $15: 111$, carnet 16 p. 2.

Figure 10 - Edgar Degas. Jockey à cheval de dos, dessin au crayon. Carnet 7, p. 27. Paris, Bibliothèque nationale de France. http://gallica.bnf.fr/ark:/12148/btv1b84387031/ f22.item.r=Degas\%20carnet7p.27, image $30: 30$, carnet 7, p. 1 . 

16, p. 64, 1859-1869. Paris, Bibliothèque nationale de France. http://gallica.bnf.fr/ ark:/12148/btv1b84387031/f22.item.r=Degas\%20carnet16 p. 64, image $18: 66$, carnet 16 p. 2.

\begin{abstract}
comme Aux courses avant le départ, 1885-1892 (Virginia Museum of Arts), Cavaliers à l'entraînement, (Moscou, musée des Beaux-Arts Pouchkine ${ }^{37}$ ), Jockeys, (Farmington, HillStead Museum ${ }^{38}$ ), chaque cheval, chaque jockey a une position différente, ce qui fait naître l'idée du mouvement dû à l'excitation qui règne au moment d'un départ de course ou quand on fait marcher les chevaux à l'aube avant l'entraînement, ou qu'ils débutent un canter. Dans l'un de ses derniers Carnets (Carnet 6), plusieurs pages sont remplies de dessins de chevaux de dos tournant vers la gauche ${ }^{39}$. Ces dessins correspondent à des modelages tels Cheval en défense ou caracolant $t^{40}$ et Cheval se cabrant $t^{41}$.
\end{abstract}

\title{
Degas peintre du mouvement
}

Degas, peintre du mouvement, en qui Paul Valéry reconnaît un « connaisseur raffiné des beautés des plus fins chevaux ${ }^{42}$ » est bien le peintre dont le regard est seul maitre pour décider de la représentation des différentes allures du cheval. Il convient de ne pas attribuer à la photographie le rôle déterminant que beaucoup lui prêtent dans l'évolution et la gestation de la peinture équestre, celle de Degas en particulier. Comme l'a souligné Waldemar George à propos de ces critiques qui en mal de véracité, raillent les peintres de chevaux qui, sans le secours de la photographie, exprimeraient de manière erronée les mouvements organiques du cheval: "La photographie croît et se développe non point sans rapports immédiats avec les autres arts de la vision mais hors de leur sphère d'influence $»^{43}$.

\section{NOTES}

1. - « Nouvelles et échos » dans le Gil Blas, 29 novembre 1881.

2. - DEBRIE, Gustave. «Les animaux dans la décoration». Revue des Arts Décoratifs, 1898, p. 287-288.

3. - Six de ces photographies sont conservées au département des Estampes et de la Photographie de la Bibliothèque nationale de France sous la cote N2.

4. - La Nature. Revue des Sciences et de leurs applications aux arts et à l'industrie. Suivi de Bulletins météorologiques de La Nature, Boîte aux Lettres, Nouvelles scientifiques. Paris : Masson, 1873-1962.

5. - Numéroté 23 à la Bibliothèque nationale de France et 31 par l'historien Théodore Reff. Les Carnets de Degas qui nous sont parvenus sont conservés au département des Estampes et de la Photographie sous la cote Res D 327d 1-29. Nous prenons dans notre étude la numérotation de la Bibliothèque nationale de France, établie quand les Carnets sont entrés dans les collections du cabinet des Estampes, en avril 1921. Ils ont été étudiés et répertoriés successivement par: LEMOISNE, P.A. « Carnets de Degas au Cabinet des Estampes ». Gazette des Beaux-Arts, 1921, p. 219-231; REFF, Théodore. The Notebooks of Edgar Degas, 2 vol. New York : Hacer Art Books, 1985 
et par BOGGS, Jean. "Degas Notebooks at the Bibliothèque nationale de France ». Burlington Magazine, 1958, p. 163-171, 196-205, 240-246.

6. - AURE, Antoine-Henri-Philippe-Léon d' (Comte). Cours d'équitation. Paris : au bureau du Journal des haras et des chasses, 1853.

7. - RAABE, Charles. « Locomotion du cheval: examen des allures selon M. H. Bouley ». Dans BOULEY, H. et REYNAL. Nouveau dictionnaire pratique de médecine. Paris : J. Dumaine, 1857.

8. - ÉLOIRE, Auguste. Le cheval, son hygiène, ses races, ses tares, sa description, sa ferrure, son signalement, ses aplombs. Paris : A-L. Guyot, s.d.

9. - RAABE, Charles. "Locomotion du cheval ». Journal des Haras, janvier-juillet 1885, p. 470-478.

10. - SOURIAU, Étienne. La Correspondance des arts. Paris : Flammarion, 1947.

11. - Dans l'ouvrage Degas et le cheval, une amitié un lieu une œuvre, à paraître Éditions Actes Sud.

12. - Dans sa thèse Degas peintre de chevaux. Vétérinaire. École vétérinaire d'Alfort. Paris : 1945.

13. - DALI, Salvador. «Hommage à Meissonier». Dans Catalogue de l'exposition du tableau de Salvador Dali la Pêche au thon à l'hôtel Meurice. Paris: Draeger, 1967, reproduit dans DALI, Salvador. Oui. Paris : Denoël/ Gonthier : 1971, p. 140.

14. - GUÉROULT, Georges. «Formes, couleurs et mouvements». Gazette des Beaux Arts, 1882, p. 178-179.

15. - THIEBAULT-SISSON, François. « Degas raconté par lui-même ». Le Temps, 11 août 1931.

16. - RAPETTI, Rodolphe. «Les cires de Gustave Moreau» dans id.. Le symbolisme. Paris: Flammarion, 2007, p. 44 et suiv.

17. - BARBOUR, Daphne S. et STURMAN, Shelley G. "Degas The sculptor and his technique". Dans LINDSAY, suzanne Glover et al. Edgar Degas Sculpture. Princeton : Princeton University Press, 2010.

18. - Carnet BnF 2, p. 225.

19. - Galerie A.A. Hébrard. Exposition des sculptures de Degas, mai-juin 1921, reproduit dans CZESTOCHOWSKI, Joseph S. et PINGEOT, Anne. Degas's Sculptures. Catalogue raisonné of the Bronzes. Memphis : Torch Press and International Arts, 2002, p. 273.

20. - REWALD, John. Degas sculpture: The complete works. Photographs by Leonardo Von Matt. New York: Harry N. Abrams, 1956.

21. - MILLARD, Charles W. The sculpture of Edgar Degas. Princeton: Princeton University Press, 1976.

22. - PINGEOT, Anne et CZESTOCHOWSKI, Joseph S., op. cit. 2002.

23. - Cheval de profil à l'arrêt, modelage en cire, Hebrard. 38, Pingeot 47. Musée d'Orsay, don Mr et Mrs Paul Mellon 1956, RF2772.

24. - LINDSAY, Suzanne Glover. "The catalogue" dans ead., op. cit. n $6 \mathrm{~A}$ et $6 \mathrm{~B} ; \mathrm{n}^{\circ} 13 ; \mathrm{n}^{\circ} 14$ et $14 \mathrm{~A}$.

25. - Le cheval au galop sur le pied gauche, tête ronde, queue en l'air, modelage en cire et bouchon, Hébrard 47, Pingeot 41. Washington National Gallery of Art, don Mr et Mrs Paul Mellon, 1999.80.11.

26. - Le Cheval au galop sur le pied droit, monté par un jockey, modelage en cire, plastiline, chiffon. Hébrard 25-35 et Pingeot 49-50. Washington National Gallery of Art, don Mr et Mme Paul Mellon, 1999.80.14 et 1999.80 .15 .

27. - Cheval au galop sur le pied gauche tournant la tête à droite et monté par un jockey, modelage en cire, Hébrard 32-36 et Pingeot 53-54. Washington, National Gallery of Art, don Mr et Mrs Paul Mellon, 1999.80.31 et 1999.80.32.

28. - ADHÉMAR, Jean. « Before the Degas bronze ». Arts News, novembre 1955, p. 34-35, 70.

29. - Les bronzes modèles achetés par Simon Norton sont conservés aujourd'hui au Norton Simon Museum à Pasadena (États-Unis).

30. - OBERTHÜR, Mariel. Degas et le cheval, une amitié, un lieu une œuvre, à paraître Éditons Actes Sud.

31. - Jockeys au départ, appelé aussi Jockeys à Epson, huile sur toile, 1862, L. 76. Collection privée. 
32. - Cheval de profil à l'arrêt, voir n. 14.

33. - Cheval pur-sang au pas, statuette originale en cire, Hébrard 66, Pingeot 38. Washington, National Gallery of Art, collection Paul Mellon.

34. - Départ d'une course devant les tribunes, essence sur papier monté sur toile, $46 \times 61 \mathrm{~cm}$ signé en bas à gauche : Degas. Paris : musée d'Orsay, don Camondo, 1911, RF 1981.

35. - Carnet 16, p. 63-69 et aussi 98 et 107.

36. - Carnet 16, p. 98, 107, 108 par exemple ou encore carnet 29, p. 11.

37. - Cavaliers à l'entraînement, pastel, 38.7 x $90.8 \mathrm{~cm}$, L. 597, Moscou, musée des Beaux-Arts Pouchkine.

38. - Jockeys, pastel sur papier, 1886, L. 596, Farmington, Hill-Stead Museum, Alfred Atmore Pope, 46-1-002.

39. - Carnet 6, p. 15 et 192-199. Carnet 7, p. 27.

40. - Cheval en défense ou caracolant, modelage en cire rouge, daté par Rewald entre 1865 et 1881, par Millard 1881-1890. Hébrard 65, Rewald, 1957, XVI, Pingeot, 40. Virginia Museum of Fine Arts, Richemond, don Paul Mellon, 1993.

41. - Cheval se cabrant, modelage en cire rouge, daté : Rewald 1865-1881, Millard 1881-1890. Hébrard 4, Rewald 13, Pingeot, 44, Washington National Gallery of Art, don Mr et Mrs Paul Mellon, 1999.

42. - VALÉRY, Paul. Degas, danse, dessin. Paris : Gallimard, 1960, p. 97.

43. - WALDEMAR, George. Photographie-Vision du monde. Paris : Arts et Métiers graphiques, $n^{\circ} 16$, 15 mars 1930.

\section{RÉSUMÉS}

Pour étudier le mouvement des allures du cheval, Degas, bien avant que ne soient publiées les photographies de Muybridge en 1878, était allé dès 1861 en Normandie, au Ménil-Hubert près du haras du Pin, chez son ami Paul Valpinçon. Là, il a fait de nombreux dessins dans des Carnets, se constituant ainsi un corpus, dans lequel il puisera pour modeler en cire des chevaux qui lui serviront pour ses tableaux et ses pastels. Cette recherche du mouvement est un élément essentiel dans l'œuvre de Degas.

Degas and the horse. To study the way horses moved, long before Muybridge's celebrated photographs were published in 1878, the painter Degas visited his friend Paul Valpinçon in Normandy, at Ménil-Hubert near the Haras du Pin stud farm. It was there, in 1861, that he drew in his notebooks, constituting a corpus from which he drew inspiration for modelling wax horses used for his pictures done in oil or pastel. This research into movement is an essential element in Degas' work.

\section{INDEX}

Keywords : horse, horse's gait, Degas, Haras du Pin, painting, pastel

Mots-clés : Degas, cheval, mouvement, peinture, pastel 


\section{AUTEUR}

\section{MARIEL OBERTHUR}

historienne, chercheur-associée CNRS/LIRE 1993-2013 mariel.oberthur@gmail.com 